\title{
The Dutch Claustrophobia Questionnaire: Psychometric properties and predictive validity
}

\author{
Ilse Van Diest ${ }^{\mathrm{a}, *}$, Dirk Smits ${ }^{\mathrm{a}, \mathrm{b}}$, Davina Decremer ${ }^{\mathrm{a}}$, Lori Maes ${ }^{\mathrm{a}}$, Laurence Claes ${ }^{\mathrm{a}}$ \\ a Department of Psychology, University of Leuven, Tiensestraat 102, B-3000 Leuven, Belgium \\ ${ }^{\mathrm{b}}$ HUB, Campus Nieuwland, Nieuwland 198, 1000 Brussels, Belgium
}

\section{A R T I C L E I N F O}

\section{Article history:}

Received 3 December 2009

Received in revised form 10 May 2010

Accepted 11 May 2010

\section{Keywords:}

Fear of suffocation

Fear of restriction

Claustrophobia fear

Exposure

Hyperventilation

\begin{abstract}
A B S T R A C T
Fear of suffocation and fear of restriction are thought to underlie claustrophobia and can be assessed with the Claustrophobia Questionnaire (CLQ; Radomsky et al., 2001). A first study tested the psychometric properties of a Dutch version of the CLQ. Students $(N=363)$ completed a Dutch translation of the CLQ and a set of other questionnaires assessing other specific fears, anxiety or depression. Results confirmed the two-factor structure and showed that the Dutch version of the CLQ has good psychometric properties. A second study tested the predictive validity of the Dutch CLQ. Participants $(N=23)$ were exposed each to nine claustrophobic situations with elements of suffocation, restriction or both. The Dutch CLQ was found to be a significant predictor of fear and respiratory reactivity during claustrophobic exposure. It can be concluded that the Dutch version of the CLQ is a reliable and valid instrument to assess claustrophobic fear.
\end{abstract}

(C) 2010 Elsevier Ltd. All rights reserved.
Claustrophobia, the fear of enclosed spaces, is a rather common specific phobia with a prevalence of $4 \%$ in the general population (Öst, 2007). Two major fear components are assumed to underlie the disorder: fear of restriction and fear of suffocation (Rachman \& Taylor, 1993). Restriction is aversive and the fear experienced by claustrophobic patients in an enclosed space may, from an evolutionary perspective, resemble the fear that animals display when they cannot escape from a (potentially) dangerous situation (Rachman, 1997). This fear of restriction is not entirely independent from the other claustrophobic fear component, fear of suffocation. Because people need a constant supply of air to stay alive, possibility of getting an insufficient amount of air is a prominent threat. Following Rachman (1997), fear of suffocation will arise when (a) people think that there is insufficient air supply in the room, (b) access to air is hampered or interrupted externally, e.g., by breathing through a mask, and (c) there is sufficient air present in the room, but the air is thought to be blocked by a physiological malfunction, for example insufficient airflow through the trachea. Also a misinterpretation of bodily signals may trigger fear of suffocation (Rachman, 1997).

The two-dimensional structure of claustrophobia was confirmed by Rachman and Taylor back in 1993 (Rachman \& Taylor,

\footnotetext{
* Corresponding author at: Research Group on Health Psychology, Psychology Department, University of Leuven, Tiensestraat 102, Box 3726, 3000 Leuven, Belgium. Tel.: +32 163260 29; fax: +32 16325923 .

E-mail address: Ilse.VanDiest@psy.kuleuven.be (I. Van Diest).
}

1993). Participants were asked how much fear they would experience in each of 36 claustrophobic situations. A principal component analysis confirmed that two factors, interpreted as fear of restriction and fear of suffocation, were underlying selfreported claustrophobic fear. Based on this study, Rachman and Taylor (1993) developed the 'The Claustrophobia Questionnaire' (CLQ). Radomsky, Rachman, Thordarson, McIsaac, and Teachman (2001) further developed the CLQ and reduced it to 26 items. They applied a principal component analysis with direct oblimin rotation, resulting in a two-factor solution with 26 items, 14 items for the suffocation scale and 12 items for the restriction scale. Both factors were moderately correlated $(r=.53)$ and accounted for $44 \%$ of the total variance in self-reported fear. Also normative data, internal consistency, discriminant validity and test-retest reliability of the 26-item CLQ were investigated. Radomsky et al. (2001) concluded that the CLQ has strong psychometric properties: it discriminates between healthy and claustrophobic individuals, it has a good internal consistency with Cronbach $\alpha$ s of $.95, .85$ and .96 for the CLQ total score, the suffocation scale and the restriction scale, respectively, and the test-retest reliability is high $(r=.89$ for the suffocation scale, $r=.77$ for the restriction scale and $r=.89$ for the total CLQ). Finally, they showed that the CLQ predicts subjective fear, bodily sensations, and apprehensive cognitions during exposure to a small enclosed space. Also several other studies have confirmed the predictive validity of the CLQ (e.g., Harris, Robinson, \& Menzies, 1999; McGlynn, Karg, \& Lawyer, 2003; McGlynn, Smitherman, Hammel, \& Lazarte, 2007; McIsaac, Thordarson, Shafran, Rachman, \& Poole, 1998). 
However, only a limited number of studies have addressed the question whether the suffocation and restriction scales of the CLQ can differentially predict fear experienced in restriction and suffocation situations, respectively. Whereas several studies show that the suffocation scale is predictive of anxious responding to laboratory challenges inducing breathlessness (e.g., Eifert, Zvolensky, Sorrell, Hopko, \& Lejuez, 1999; Eke \& McNally, 1996; Rassovsky, Kushner, Schwarze, \& Wangensteen, 2000; Shipherd, Beck, \& Ohtake, 2001) less is known on the specific predictive validity of the restriction scale. In a study by Van Diest et al. (2005) participants completed a Dutch ad-hoc translation of six items loading high on the restriction scale and six other items loading high on the suffocation scale of the English version of the CLQ (Radomsky et al., 2001). Next, participants had to imagine three standardized fear scripts, one depicting a situation with aspects of both restriction and risk of suffocation, one referring to restriction only, and one referring to neither claustrophobic fear components. Interestingly, participants' scores on the restriction scale predicted subjective fear, but not respiratory reactivity to imagined restriction; scores on the suffocation scale were not related to self-reported fear or respiratory reactivity during the imagined exposure.

The present studies aimed to (a) develop a validated Dutch version of the CLQ and (b) further explore the predictive validity of the CLQ. In particular, we aimed to address the question to what extent the restriction and the suffocation scales of the Dutch CLQ allow for prediction of actual fear and respiratory reactivity in claustrophobic situations with or without restriction and/or risk of suffocation.

\section{Study 1: Validation of the Dutch CLQ}

The first study aimed to develop a Dutch version of Radomsky et al.'s (2001) Claustrophobia Questionnaire.

\subsection{Method}

\subsubsection{Participants}

Participants were 371 undergraduate, Dutch-speaking students (282 women) aged 18-23 years old. They all received course credit in return for their participation. All participants provided an informed consent. The study was approved by the Ethics Committee of the Department of Psychology (University of Leuven, Belgium).

\subsubsection{Procedure}

The English CLQ was translated and back-translated several times until agreement was reached among three Dutch-speaking persons with a profound knowledge of English. Next, during two 1$h$ group sessions one week apart, participants completed the Dutch translation of the CLQ once, as well as a series of other questionnaires (see below).

\subsubsection{Measures}

1.1.3.1. The Claustrophobia Questionnaire (CLQ; Radomsky et al., 2001). Participants had to rate the 26 claustrophobic items on a five-point scale ranging from 0 (not at all anxious) to 4 (extremely anxious).

1.1.3.2. The Beck Depression Inventory-II-NL (BDI-II-NL; van der Droes, 2002). The BDI-II (Beck, Steer, \& Brown, 1996) is a frequently used questionnaire to measure self-experienced cognitive and somatic symptoms of depression. The Dutch translation of the BDI-II, the BDI-II-NL has good psychometric qualities. The internal consistency is high with Cronbach $\alpha$ s of .92 and .88 for a patient population and a control population, respectively. Also the test- retest reliability $(r=.82)$ and the convergent validity are good (van der Droes, 2002). Cronbach $\alpha$ in our sample of students was .88 .

1.1.3.3. The Dutch NEO Five-Factor Inventory (NEO-FFI-NL; Hoekstra, Ormel, \& De Fruyt, 1996). The Dutch version of the NEO-FFI (NEO-FFI-NL) contains 60 questions assessing the big five personality traits, Neuroticism (NEO-FFI-NL-N), Extraversion, Openness to experience, Altruism and Conscientiousness. For the present study, only scores on Neuroticism were investigated. This subscale obtained a Cronbach $\alpha$ of .86 in our sample.

1.1.3.4. Spielberger's State-Trait Anxiety Inventory (STAI; Spielberger, 1983). Barenes, Harp, and Jung (2002) reviewed 816 papers in which the STAI was used to measure trait and state anxiety. They report a mean internal consistency of .91 and .89 for the state and trait subscales, respectively. The mean of the test-retest reliability was .70 for the state and .88 for the trait subscale. van der Ploeg (2000) translated the STAI into Dutch. He reported Cronbach $\alpha$ s of .92 (state) and .90 (trait). Cronbach $\alpha$ in our student sample was .82

1.1.3.5. Anxiety Sensitivity Index-3 (ASI-3; Taylor et al., 2007). The ASI-3 is a multidimensional measurement of anxiety sensitivity. It encompasses three factors: Physical Concerns, Cognitive Concerns, and Social Concerns. The validation study of the Dutch version demonstrated a good validity of the factor structure, as well as a good internal consistency for each of the three scales (Cronbach $\alpha \mathrm{s}$ were .80 for Physical Concerns, .81 for Cognitive Concerns, and .76 for Social Concerns; Taylor et al., 2007). Cronbach $\alpha$ for the total scale in our student sample was .83 .

1.1.3.6. Fear of Pain Questionnaire-III (FPQ-III; McNeil E' Rainwater, 1998). The FPQ-III consists of 30 items related to the fear of severe pain, minor pain, and medical pain. These factors accounted for $51 \%$ of total variance. The scales of the questionnaire are internally consistent with Cronbach $\alpha$ s of .88, .87, .87, and .92 for the three subscales and the total scale, respectively. The test-retest reliability is also good with correlations of $.69, .73, .76$, and .74 for the three subscales and the total scale, respectively (McNeil \& Rainwater, 1998). Roelofs, Peters, Deutz, Spijker, and Vlaeyen (2005) translated this questionnaire into Dutch. They also found evidence for the three subscales, severe pain, minor pain, and medical pain. For a sample of first-year college students, they found Cronbach $\alpha \mathrm{s}$ $.93, .88, .86$, and .88 for the total FPQ-III and the subscales, respectively. For a sample of first-year and higher-year college students they found Cronbach $\alpha$ s $91, .89, .82$, and .85 for the total FPQ-III and the subscales, respectively. They reported a moderate to good test-retest stability and a good convergent and predictive validity. Cronbach $\alpha$ in our sample of students was .92 for the total FPQ-III.

1.1.3.7. The Claustrophobia Scale(CS; Öst, 2007). The claustrophobia scale consists of two scales: one scale measuring anxiety (CSA) and one scale assessing avoidance behavior (CSB). We translated both scales ourselves, because no Dutch validated version is available. The English SC has a high reliability with Cronbach $\alpha$ s .97 and .81 for the anxiety and avoidance subscales, respectively. In our translated Dutch version, the anxiety subscale had a Cronbach $\alpha .89$; Cronbach $\alpha$ for the avoidance subscale was .80 .

1.1.3.8. Fear Survey Schedule-III (FSS-III; Wolpe E Lang, 1964). This questionnaire assesses specific fears. Psychometric properties for a Dutch version of the FSS-III have been described by Arindell (1980) and are good, with a Cronbach $\alpha$ of .95. Cronbach $\alpha$ for the total scale in our sample was .97. Three items of the FSS-III involve claustrophobic fear (FSS-III-CL), such as fear of 'crowds,' 'being in an elevator,' and 'enclosed spaces.' The other 73 items are classified 
Table 1

Fit indices for the confirmatory factor analyses on the items of the Dutch Claustrophobia Questionnaire.

\begin{tabular}{|c|c|c|c|c|c|c|c|}
\hline & $\mathrm{df}$ & Satorra-Bentler $\mathrm{Chi}^{2}$ & AIC & CFI & RMSEA & NNFI & NFI \\
\hline One-factor model & 299 & 1184.35 & 1288.35 & .94 & .09 & .93 & .91 \\
\hline Two-factor model & 298 & 697.34 & 803.34 & .97 & .06 & .97 & .95 \\
\hline
\end{tabular}

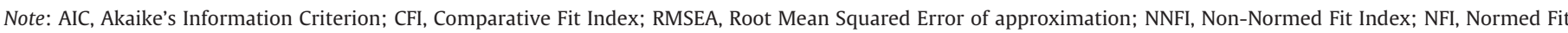
Index.

in six categories all measuring another specific fear: 'animal' (FSSIII-A), 'social or interpersonal' (FSS-III-S), 'tissue damage, illness and death, and their associations' (FSS-III-T), 'noises' (FSS-III-N), 'other classical phobias' (FSS-III-O), and 'miscellaneous' (FSS-III-M).

\subsubsection{Data analyses}

As we have a clear hypothesis about the factorial structure of the CLQ (i.e., two-factorial), confirmatory factor analyses (CFAs) were performed with LISREL 8.71 (Jöreskog \& Sörbom, 2004). To test whether the power of the two-factor model of Radomsky et al. (2001) was large enough to reject a more parsimonious one-factor alternative, we fitted an additional one-factor model (Bentler, 2007), assuming one general underlying claustrophobia factor. For the two-factor model, each item loaded on its corresponding factor: restriction or suffocation, as specified in Radomsky et al. (2001). Both latent factors were allowed to correlate.

Because our indicators are categorical variables, CFA models were estimated using a Robust Weighted Least Square estimation method, on the polychoric correlations, weighted by the asymptotic variances (Flora \& Curran, 2004). Fit was assessed by multiple criteria: Satorra-Bentler scales Chi-square values for absolute fit, comparative fit index (CFI) for fit relative to a null model and, additionally, the root mean squared error of approximation (RMSEA). Criteria for fit were defined according to Hu and Bentler (1999) as CFI $>.97$ and RMSEA $<.06$. Fit of nested models is compared with Chi-square difference test.
To investigate internal consistency, Cronbach $\alpha$ s were calculated for the Dutch CLQ, the restriction scale, and the suffocation scale.

In order to study the convergent validity, correlations were calculated between CLQ scores and scores on (subscales of) other questionnaires tapping claustrophobic fears (SC and FSS-III-CL).

The divergent validity of the Dutch CLQ was investigated using t-tests, comparing the correlations of the Dutch CLQ with other measures of claustrophobia (SC, FSS-III-CL) with those of the Dutch CLQ with questionnaires assessing other fears (FSS-III-A, the FSSIII-S, the FSS-III-T, the FSS-III-N, the FSS-III-O, the FSS-III-M FPQ-III, ASI-3), depression (BDI-II-NL), trait anxiety (STAI), and Neuroticism (NEO-II-NL-N).

\subsection{Results}

\subsubsection{Factor structure}

The two-factor model obtained a better fit than the one factor model (Chi-Square difference $=487.01 ; \mathrm{df}=1 ; p<.001)$. Moreover, the two-factor model obtained a reasonable fit to the data (see Table 1). Both factors correlated .68. Factor loadings of the twofactor model are presented in Table 2.

\subsubsection{Normative data}

Means and standard deviations of the Dutch CLQ items can be found in Table 2.

Table 2

Means (SDs) for the items of the Dutch CLQ $(N=362)$ and factor loadings for the restriction and the suffocation scale.

\begin{tabular}{|c|c|c|c|c|}
\hline \multirow[t]{2}{*}{ Item } & \multicolumn{2}{|c|}{ Factor loading } & \multirow[t]{2}{*}{$M$} & \multirow[t]{2}{*}{ SD } \\
\hline & $\mathrm{R}$ & $\mathrm{S}$ & & \\
\hline Standing for $15 \mathrm{~min}$ in a straitjacket & .69 & & 1.41 & 1.12 \\
\hline Having your legs tied to an immovable chair & .73 & & .94 & .93 \\
\hline Tied up with hands behind back for $15 \mathrm{~min}$ & .79 & & .82 & .92 \\
\hline Handcuffed for $15 \mathrm{~min}$ & .74 & & .78 & .97 \\
\hline Locked in a small DARK room without windows for $15 \mathrm{~min}$ & .77 & & 1.86 & 1.18 \\
\hline $\begin{array}{l}\text { Lying in a tight sleeping bag enclosing legs and arms, tied at the neck, } \\
\text { unable to get out for } 15 \mathrm{~min}\end{array}$ & .70 & & 1.44 & 1.22 \\
\hline Lying in the trunk of a car with air flowing through freely for $15 \mathrm{~min}$ & .77 & & 1.77 & 1.28 \\
\hline Caught in tight clothing and unable to remove it & .57 & & .95 & .96 \\
\hline Head First into a zipped up sleeping bag, able to leave whenever you wish & .56 & & .88 & 1.06 \\
\hline Locked in a small WELL-LIT room without windows for $15 \mathrm{~min}$ & .70 & & .50 & .86 \\
\hline In a crowded train which stops between stations & .51 & & .53 & .82 \\
\hline In a public washroom and the lock jams & .59 & & 1.52 & 1.06 \\
\hline Swimming while wearing a nose plug & & .44 & .29 & .63 \\
\hline Working under a sink for $15 \mathrm{~min}$ & & .70 & .24 & .59 \\
\hline Standing in an elevator on the ground floor with the doors closed & & .70 & .40 & .79 \\
\hline Trying to catch your breath during vigorous exercise & & .55 & .31 & .59 \\
\hline Having a bad cold and finding it difficult to breath through your nose & & .58 & .31 & .61 \\
\hline Snorkelling in a safe practice tank for $15 \mathrm{~min}$ & & .51 & .43 & .77 \\
\hline Using an oxygen mask & & .60 & .58 & .72 \\
\hline Lying on a bottom bunk bed & & .59 & .14 & .45 \\
\hline $\begin{array}{l}\text { Standing in the middle of the third row at a packed concert realizing that } \\
\text { you will be unable to leave until the end }\end{array}$ & & .55 & .49 & .83 \\
\hline In the centre of a full row at a cinema & & .47 & .10 & .36 \\
\hline Working under a car for $15 \mathrm{~min}$ & & .70 & .50 & .78 \\
\hline At the furthest point from an exit on a tour of an underground mine shaft & & .64 & 1.02 & 1.04 \\
\hline Lying in a sauna for $15 \mathrm{~min}$ & & .53 & .30 & .68 \\
\hline Waiting for $15 \mathrm{~min}$ in a plane on the ground with the doors closed & & .58 & .28 & .67 \\
\hline
\end{tabular}

Note: S, suffocation scale of the Dutch CLQ; R, restriction scale of the Dutch CLQ. 
Table 3

Correlations between the Dutch CLQ and other questionnaires $(N=362)$.

\begin{tabular}{ll}
\hline & Dutch CLQ \\
\hline FSS-III-CL & $.61^{*}$ \\
CSA & $.73^{*}$ \\
CSB & $.55^{*}$ \\
BDI-II-NL & $.35^{*}$ \\
NEO-FFI-NL-N & $.35^{*}$ \\
STAI & $.39^{*}$ \\
ASI-3 & $.39^{*}$ \\
FPQ-III & $.41^{*}$ \\
FSS-III-A & $.37^{*}$ \\
FSS-III-S & $.49^{*}$ \\
FSS-III-T & $.46^{*}$ \\
FSS-III-N & $.42^{*}$ \\
FSS-III-O & $.54^{*}$ \\
FSS-III-M & $.46^{*}$ \\
\hline
\end{tabular}

Note: CLO Claustrophobia Questionnaire; FSS-III-CL claustrophobia subscale of the Fear Survey Schedule-III; CSA, anxiety subscale of the Claustrophobia Questionnaire; CSB, avoidance behavior subscale of the Claustrophobia Questionnaire; BDI-II-NL, Dutch version of the Beck Depression Inventory-II; NEO-FFI-NL-N, Neuroticism subscale of the Dutch NEO Five-Factor Inventory; STAI, Spielberger's State-Trait Anxiety Inventory; ASI3. Anxiety Sensitivity Index-3; FPQ-III, Fear of Pain Questionnaire-III; FSS-III-A, animal subscale of the Fear Survey Schedule-III; FSS-III-S, social or interpersonal subscale of the Fear Survey Schedule-III; FSS-III-T, tissue damage. FSS-III-N, noises subscale of the Fear Survey Schedule-III; FSS-III-O, other classical phobias subscales of the Fear Survey Schedule-III; FSS-III-M, miscellaneous subscale of the Fear Survey Schedule-III.

$* p<.05$.

\subsubsection{Internal consistency}

Cronbach $\alpha$ was .90 for the Dutch CLQ. For the subscales of restriction and suffocation scale, Cronbach $\alpha$ s were .88 and .80 , respectively.

\subsubsection{Convergent validity}

Table 3 displays the correlations between the Dutch CLQ and 3 other measures of self-reported claustrophobic fear. The Dutch CLQ correlated strongly with the FSS-III-CL $(r=.61, p<.05)$, the CSA $(r=.73, p<.05)$ and the CSB $(r=.55, p<.05)$, indicating a good convergent validity.

\subsubsection{Divergent validity}

Generally, correlations between the Dutch CLQ and other tools assessing claustrophobic fear were significantly higher than the correlations of the Dutch CLQ with questionnaires assessing fears other than claustrophobia or with general measures of depression or anxiety (see Table 3 ).

More specifically, the Dutch CLQ correlated higher with the FSSIII-CL than with the BDI-II-NL, $t(304)=5.11, p<.05$, the NEO-FFINL-N, $t(304)=4.85, p<.05$, the STAI, $t(304)=4.17, p<.05$, the ASI-3, $t(304)=4.29, p<.05$, the FPQ-III, $t(304)=3.74, p<.05$, the FSS-III-A, $t(304)=4.40, p<.05$, the FSS-III-S, $t(304)=2.66, p<.05$, the FSS-III-T, $t(304)=3.13, p<.05$, the FSS-III-N, $t(304)=4.08, p<.05$, the FSS-III$\mathrm{O}, t(304)=1.69, p<.05$, and the FSS-III-M, $t(304)=3.13, p<.05$.

Similarly, the Dutch CLQ correlated more strongly with the CSA than with the following questionnaires: BDI-II-NL, $t(304)=8.32$, $p<.05$, NEO-FFI-NL-N, $t(304)=8.06, p<.05$, STAI, $t(304)=7.55$, $p<.05$, ASI- $3, t(304)=7.95, p<.05$, FPQ-III, $t(304)=7.46, p<.05$, FSSIII-A, $t(304)=8.30, p<.05$, FSS-III-S, $t(304)=6.51, p<.05$, FSS-III-T, $t(304)=7.03, p<.05$, the FSS-III-N, $t(304)=8.15, p<.05$, the FSS-III$\mathrm{O}, t(304)=6.30, p<.05$, and the FSS-III-M, $t(304)=7.17, p<.05$.

In the same vein, correlations between the Dutch CLQ and the CSB were significantly higher than correlations between the
Dutch CLQ and the BDI-II-NL, $t(304)=3.39, p<.05$, the NEO-FFINL-N, $t(304)=3.39, p<.05$, the STAI, $t(304)=2.79, p<.05$, the ASI-3, $t(304)=2.93, p<.05$, the FPQ-III, $t(304)=2.57, p<.05$, the FSS-III-A $t(304)=3.27, p<.05$, the FSS-III-T, $t(304)=1.80, p<.05$, the FSS-III$\mathrm{N}, t(304)=2.45, p<.05$, and the FSS-III-M, $t(304)=1.77, p<.05$. The correlation between the Dutch CLQ and the SCB did not significantly differ from those between the Dutch CLQ and the FSS-III-O and between the Dutch CLQ and the FSS-III-S.

\section{Study 2: Predictive validity of the Dutch CLQ}

The aim of the second study was to investigate to what extent the Dutch CLQ and its subscales predict self-reported fear and respiratory reactivity during actual exposure to different claustrophobic situations. To this end, participants were exposed to nine claustrophobic situations that varied with respect to the presence or absence of elements of restriction and risk of suffocation. We hypothesized that (a) the restriction scale would be the better predictor for reactivity to restriction situations without elements referring to risk of suffocation, (b) the suffocation scale would be the better predictor for reactivity to situations referring to risk of suffocation but without restriction, and (c) the total score on the Dutch CLQ would predict fearful responding to all types of claustrophobic situations.

\subsection{Method}

\subsubsection{Participants}

Based on the factor scores on fear of suffocation and fear of restriction, which were calculated for each of the 362 participants from study 1,41 students scoring high for restriction or suffocation (not both) were invited for the experiment in a first wave. Because only 8 participants could be recruited this way, all 362 participants from study 1 were invited in a second wave. A total 23 students volunteered to participate in return for course credit or a fee of 7 Euro. Three of them were men. The study was approved by the ethical commission of Psychology Department of the University of Leuven.

\subsubsection{Measures}

2.1.2.1. Subjective measures. Before entering each claustrophobic situation, participants rated how fearful they expected to be in that particular situation (PRE). After exposure, participants rated their average fear level during the exposure (POST) and during the most fearful moment (PEAK). Each of these questions was rated on an 11point Likert scale ranging from 0 (not at all fearful) to 10 (extremely fearful).

2.1.2.2. Physiological measures. Cardio-respiratory activity was measured continuously with the LifeShirt System (VivoMetrics, Inc., Ventura, CA), an ambulatory system using inductive plethysmography and ECG. End-tidal $\mathrm{CO}_{2}$ was monitored using a nasal $\mathrm{CO}_{2}$-sampling cannula connected to an Oridion Microcap Handheld Capnograph, with a sampling flow rate of $50 \mathrm{ml} / \mathrm{min}$. The monitor uses Microstream non-dispersive infrared (NDIR) spectroscopy to continuously measure the percentage of $\mathrm{CO}_{2}$ at the end of an expiration $\left(\mathrm{FetCO}_{2}\right) . \mathrm{FetCO}_{2}$ is a good estimate of the level of $\mathrm{CO}_{2}$ in the blood (Gardner, 1996). The $\mathrm{CO}_{2}$-signal was recorded using the Vivologic software. End-tidal values for each breath were extracted off-line with PSychoPHysiological Analysis (PSPHA - De Clerck, Verschuere, Crombez, \& De Vlieger, 2006), a modular script-based program which we further developed to perform such parameter extraction. All waveforms were visually inspected off-line and technical abnormalities and artifacts were eliminated using the PSPHA software.

The present paper will only report findings on $\mathrm{FetCO}_{2}$, because of two reasons. First, in contrast to parameters of heart rate and 
timing and volume components of the respiratory cycle, FetCO varies not as a function of muscle activity and body position, for which the nine claustrophobic situations were not equalized. Because blood gases of healthy humans are kept rather constant, even under changing metabolic conditions, the end-tidal $\mathrm{CO}_{2}$ level is not expected to differ between situations where participants have to stand, to sit down or to walk from one room to another. In addition, $\mathrm{FetCO}_{2}$ is the most relevant indicator of hyperventilation, a situational respiratory stress response in which more $\mathrm{CO}_{2}$ is breathed out than is actually being produced by the body, leading to a decreased pressure of $\mathrm{CO}_{2}$ in the blood and in the exhaled air (Van Diest et al., 2001).

\subsubsection{Procedure}

After providing their informed consent, participants put on the lifeshirt and the nasal cannula. Following this, a $10 \mathrm{~min}$ baseline registration of resting heart rate, respiration and $\mathrm{FetCO}_{2}$ was performed. Next, the experimenter explained to the participants that they would be asked to enter several particular situations and that they would be asked to rate on a scale from 0 to 10 (1) how fearful they expected themselves to be in that situation (PRE), (2) the maximum fear level they had experienced during actual exposure to the situation (PEAK), and (3) their average level of fear during the actual exposure (POST).

The nine claustrophobic situations were composed by crossing two independent variables with three levels each. The first variable ('Situation') referred to whether the claustrophobic exposure involved (a) standing in a large room ('standing large'), (b) sitting on a chair in the large room ('sitting large'), or (c) standing in a small room ('standing small'). The second independent variable referred to the claustrophobic fear that the situation intended to evoke: (a) only restriction (' $R$ '), (b) only suffocation (' $S$ '), or (c) both restriction and suffocation ('RS'). Both variables were fully crossed, yielding nine situations. Nine presentation orders for these nine situations were created in such a way that the same situation (standing large/sitting large/standing small) and the same claustrophobic component (RS/R/S) were never presented twice in a row. Participants were counterbalanced across these nine presentation orders. Each situation lasted for $2 \mathrm{~min}$, but participants were not informed on this.

In situation 'standing large-S' participants had to stand in a large room $(5.10 \times 6.85 \mathrm{~m}, 3 \mathrm{~m}$ high $)$ while wearing a face mask covering their nose and mouth and wearing a shawl around their neck.

In situation 'standing large-R' participants had to stand up in a locked dark closet that was placed in the large room. The closet had a surface of $1 \times 4 \mathrm{~m}$, was $1.85 \mathrm{~m}$ high and had 30 visible holes in the back of the closet with a diameter of $.5 \mathrm{~cm}$. These holes aimed to suggest a supply of 'fresh air' while the participant remained in the closet.

In situation 'standing large-RS' participants had to stay in a similar, locked dark closet without such holes, suggesting that no fresh air was available.

In situation 'sitting large-R' participants were sitting in the large room with their hands handcuffed behind their back and with their feet tied to the chair with a rope. In situation 'sitting large-S' participants were wearing a mask covering their nose and mouth while sitting on a chair in the large room.

In situation 'sitting large-RS' participants were sitting down in a large room while tied up to the chair in a similar ways as in situation 'sitting large-R.' In addition, they were wearing a face mask covering their nose and mouth.

In situation 'standing small-R' participants were locked up in a dark, small room. A fan provided fresh air in the room. The room's surface was $220 \times 90 \mathrm{~cm}$ and had a small kitchenette in the back; the height of the room was $270 \mathrm{~cm}$.
In situation 'standing small-S' participants were standing in the same small room, but now the room was unlocked, the fan was removed and they were wearing a neck supportive bandage.

In situation 'standing small-RS' participants were wearing a neck bracket and were locked up in the small room, without the fan.

Before entering each of the nine situations, participants received both oral and written information about the specificities of situation. For example, in situation 'standing-RS' participants were told "In a moment, you will enter this closet and we will lock you in with this key. Next, we will leave the room. After a while, we will re-enter the room to unlock the closet, so you can come out." The same information was written down on a sheet, which they were asked to read after the explanation by the experimenter. Then, participants were asked to rate how much fear they expected to experience during the situation (PRE question). Following this, participants entered the situation for two minutes without knowing the duration of the exposure. After the exposure, participants completed the other fear ratings (PEAK and POST). This procedure was repeated for each of the other eight situations. After the experiment the LifeShirt was disconnected and participants changed back into their own clothes. They received their course credit or money, were debriefed and thanked for their participation.

\subsubsection{Analyses}

2.1.4.1. Subjective measurements. In order to reduce the amount of dependent variables, a sum of the three fear scores (PRE, PEAK, and POST) was calculated for each situation and each participant. In a next step, these scores were averaged across the RS, R, and $S$ situations for each participant, yielding three fear indexes per participant: fear in situations characterized by both claustrophobic components (fear RS), fear in situations with only risk of suffocation (fear S), and fear in situations with only the restriction component (fear R). These fear indexes were correlated with each individual's scores on the Dutch CLQ and its subscales (fear of restriction and fear of suffocation), as well as with the(sub)scores on all other questionnaires (FSS-III-CL, SCA, SCB, BDI-II-NL, NEO-II-NL-N, STAI, ASI-3, FPQ-III, FSS-III-A, FSS-III-S, FSS-III-T, FSS-III-N, FSS-III-O, and FSS-III-M).

2.1.4.2. Fractional end-tidal $\mathrm{CO}_{2}\left(\mathrm{FetCO}_{2}\right)$. FetCO $\mathrm{CO}_{2} \mathrm{~S}$ of each breath were averaged across each $2 \mathrm{~min}$ exposure for each participant. The mean $\mathrm{FetCO}_{2}$ during the 10 min baseline was subtracted from these mean $\mathrm{FetCO}_{2} \mathrm{~s}$ during each of the nine exposures, yielding an index of reactivity in $\mathrm{FetCO}_{2}$ for each situation. These reactivity scores in $\mathrm{FetCO}_{2}$ were averaged across the three RS situations, the three R situations and the three $\mathrm{S}$ situations per participant. In a final step, these reactivity scores in $\mathrm{FetCO}_{2}$ were correlated with each individual's scores on the Dutch CLQ and its subscales (fear of restriction and fear of suffocation), as well as with the (sub)scores of all other questionnaires (FSS-III-CL, SCA, SCB, BDI-II-NL, NEO-II-NL-N, STAI, ASI-3, FPQ-III, FSS-III-A, FSS-III-S, FSS-III-T, FSS-III-N, FSS-III-O, and FSS-III-M).

\subsection{Results}

\subsubsection{Self-reported fear}

Mean scores of the participants on the Dutch CLQ and its subscales as assessed in study 1 were: $M=23.24(\mathrm{SD}=11.83)$ for the total score, $M=15.15(S D=8.83)$ for the restriction scale and $M=7.55(\mathrm{SD}=6.16)$ for the suffocation scale. The correlation between both scales was not significant in this sample $(r=.23$, $N=21$, see Fig. 1 for a scatter plot showing how participants were distributed across both subscales).

Table 4 displays the mean self-reported fear scores and standard deviations for the nine claustrophobic situations. 


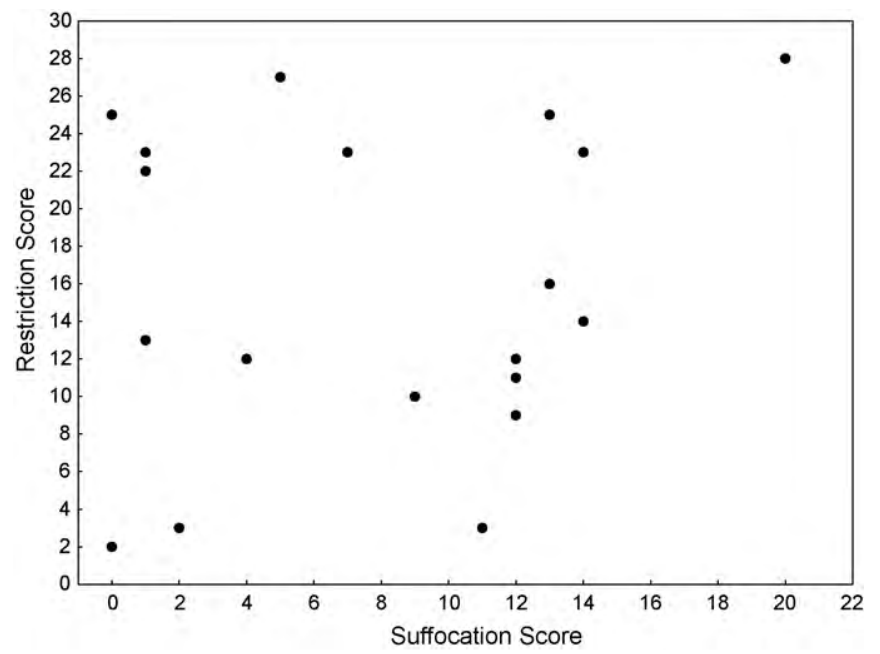

Fig. 1. Scatter plot of the suffocation and restriction scores of the participants in study 2 .

Table 5 shows the correlations between self-reported fear to actual exposure and the questionnaire data. Fear RS, fear R and fear $S$ did not correlate with any of the questionnaires not assessing claustrophobic fear. In contrast with this, several significant correlations were observed between the Dutch CLQ or its subscales on the one hand, and self-reported fear during actual claustrophobic exposure on the other hand. More specifically, the total score on the Dutch CLQ showed significant, moderate, positive correlations with Fear RS, Fear R and Fear S. The restriction subscale of the Dutch CLQ was moderately correlated with Fear S and Fear R, but not with Fear RS. The correlations of the suffocation subscale of the Dutch CLQ with Fear RS, Fear R and Fear S did not reach significance. Also the other measures of self-reported claustrophobic fear (FSS-III-CL, CSA, CSB) showed significant, moderately strong correlations with Fear RS, Fear R and Fear S. An exception to this was the non-significant correlations between FSS-III-CL and Fear RS.

\subsection{2. $\mathrm{FetCO}_{2}$}

Mean changes in $\mathrm{FetCO}_{2}$ in response to the three types of claustrophobic situations were: $M=-.10(\mathrm{SD}=.14)$ for RS situations, $M=-.08(\mathrm{SD}=.15)$ for $\mathrm{R}$ situations and $M=.01(\mathrm{SD}=.17)$ for suffocation situations.

Table 6 displays correlations between changes in $\mathrm{FetCO}_{2}$ during exposure to claustrophobic situations of each type and the questionnaire data. Higher scores on the Dutch CLQ were significantly associated with a stronger decrease in $\mathrm{FetCO}_{2}$ during actual exposure to situations with both elements of suffocation and restriction (RS). The correlations between the subscales on the one hand, and

Table 4

Mean (SDs) self-reported fear in response to nine claustrophobic situations $(N=23)$.

\begin{tabular}{lll}
\hline Situations & Fear & \\
\cline { 2 - 3 } & $M$ & SD \\
\hline Standing large-RS & 14.93 & 7.23 \\
Standing large-R & 13.17 & 7.71 \\
Standing large-S & 13.80 & 6.32 \\
Sitting large-RS & 13.96 & 7.81 \\
Sitting large-R & 9.93 & 5.73 \\
Sitting large-S & 9.24 & 6.67 \\
Standing small-RS & 11.34 & 6.49 \\
Standing small-R & 11.30 & 6.22 \\
Standing small-S & 10.22 & 6.39 \\
\hline
\end{tabular}

Note: RS, situations with restriction and risk of suffocation; R, situations with restriction and without risk of suffocation; $S$, situations with risk of suffocation and without restriction.
Table 5

Correlations between experienced fear in response to claustrophobic exposure and the questionnaire scores $(N=21)$

\begin{tabular}{lccc}
\hline & Fear RS & Fear S & Fear R \\
\hline CLQ & $.47^{*}$ & $.53^{*}$ & $.55^{*}$ \\
S & .26 & .31 & .28 \\
R & .42 & $.45^{*}$ & $.52^{*}$ \\
FSS-III-CL & .39 & $.51^{*}$ & $.55^{*}$ \\
CSA & $.55^{*}$ & $.50^{*}$ & $.60^{*}$ \\
CSB & $.48^{*}$ & $.48^{*}$ & $.49^{*}$ \\
BDI-II-NL & & & .23 \\
NEO-FFI-NL-N & .08 & .23 & .26 \\
STAI & .08 & .21 & .16 \\
ASI-3 & -.01 & .21 & .03 \\
FPQ-III & -.1 & .03 & .09 \\
FSS-III-A & .05 & .15 & .23 \\
FSS-III-S & .13 & .19 & .16 \\
FSS-III-T & .06 & .10 & .11 \\
FSS-III-N & .03 & .18 & .23 \\
FSS-III-O & .07 & .07 & .22 \\
FSS-III-M & .06 & .19 & .02 \\
\hline
\end{tabular}

Note: RS, situations with restriction and risk of suffocation; S, situations with risk of suffocation only; R, situations with restriction only; FSS-III-CL, claustrophobia subscale of the Fear Survey Schedule-III; CSA, anxiety subscale of the Claustrophobia Questionnaire; CSB, avoidance behavior subscale of the Claustrophobia Questionnaire; BDI-II-NL, Dutch version of the Beck Depression Inventory-II; NEO-FFI-NL-N, Neuroticism subscale of the Dutch NEO Five-Factor Inventory; STAI, Spielberger's State-Trait Anxiety Inventory; ASI-3, Anxiety Sensitivity Index-3; FPQ-III, Fear of Pain Questionnaire-III; FSS-III-A, animal subscale of the Fear Survey Schedule-III; FSS-III-S, social or interpersonal subscale of the Fear Survey Schedule-III; FSS-III-T, tissue damage. FSS-III-N, noises subscale of the Fear Survey Schedule-III; FSS-III-O other classical phobias subscales of the Fear Survey Schedule-III; FSS-III-M, miscellaneous subscale of the Fear Survey Schedule-III. $* p<.05$.

Table 6

Correlations between reactivity in $\mathrm{FetCO}_{2}$ and questionnaires scores $(N=20)$.

\begin{tabular}{llll}
\hline & $\mathrm{dFetCO}_{2} \mathrm{RS}$ & $\mathrm{dFetCO}_{2} \mathrm{~S}$ & $\mathrm{dFetCO}_{2} \mathrm{R}$ \\
\hline $\mathrm{CLQ}$ & $-.47^{*}$ & -.32 & -.37 \\
$\mathrm{~S}$ & -.27 & -.24 & -.28 \\
$\mathrm{R}$ & -.42 & -.36 & -.21 \\
$\mathrm{FSS}-$ III-CL & -.31 & -.31 & -.41 \\
$\mathrm{CSA}$ & -.27 & -.24 & -.38 \\
$\mathrm{CSB}$ & -.25 & -.27 & -.15 \\
BDI-II-NL & .13 & .01 & .05 \\
NEO-FFI-NL-N & -.21 & -.02 & .10 \\
STAI & -.01 & -.07 & -.02 \\
ASI-3 & .04 & .03 & .11 \\
FPQ-III & -.11 & -.18 & -.05 \\
FSS-III-A & -.30 & -.39 & -.38 \\
FSS-III-S & .01 & -.01 & .07 \\
FSS-III-T & -.26 & -.26 & -.21 \\
FSS-III-N & -.18 & -.20 & -.22 \\
FSS-III-O & -.20 & -.33 & -.27 \\
FSS-III-M & .07 & -.06 & .08 \\
\hline
\end{tabular}

Note: $\mathrm{dFetCO}_{2}$, reactivity in Fractional End-tidal $\mathrm{CO}_{2} ; \mathrm{RS}$, situations with restriction and risk of suffocation; $S$, situations with risk of suffocation only; R, situations with restriction only; FSS-III-CL, claustrophobia subscale of the Fear Survey Schedule-III; CSA, anxiety subscale of the Claustrophobia Questionnaire; CSB, avoidance behavior subscale of the Claustrophobia Questionnaire; BDI-II-NL, Dutch version of the Beck Depression Inventory-II; NEO-FFI-NL-N, Neuroticism subscale of the Dutch NEO Five-Factor Inventory; STAI, Spielberger's State-Trait Anxiety Inventory; ASI-3, Anxiety Sensitivity Index-3; FPQ-III, Fear of Pain Questionnaire-III; FSS-III-A, animal subscale of the Fear Survey Schedule-III; FSS-III-S, social or interpersonal subscale of the Fear Survey Schedule-III; FSS-III-T, tissue damage. FSS-III-N, noises subscale of the Fear Survey Schedule-III; FSS-III-O, other classical phobias subscales of the Fear Survey Schedule-III; FSS-III-M, miscellaneous subscale of the Fear Survey ScheduleIII. ${ }^{*} p<.05$. 
reactivity in $\mathrm{FetCO}_{2}$ during actual exposure on the other hand, were not significant. A similar pattern of results was present for the correlations between other questionnaires measuring claustrophobia (CSA, CSB, FSS-III-CL) and reactivity in $\mathrm{FetCO}_{2}$ during actual exposure.

Reactivity in $\mathrm{FetCO}_{2}$ during actual claustrophobic exposure did not correlate with scores on any of the questionnaires not assessing claustrophobic fear.

\section{Discussion}

A first study aimed to validate the Dutch version of the CLQ. In accordance with the original English version of the CLQ (Rachman \& Taylor, 1993; Radomsky et al., 2001), the Dutch CLQ consists of two factors, fear of restriction and fear of suffocation. The internal consistency of the Dutch CLQ is acceptable and comparable to those found for the English CLQ (Radomsky et al., 2001), confirming that it is a reliable instrument.

Furthermore, our results also showed that the Dutch CLQ has a good divergent and convergent validity. Whereas it correlates strongly with other self-report measures of claustrophobia, the correlations with questionnaires measuring specific fears other than claustrophobia, depression, and dispositional anxiety or neuroticism were substantially lower. Therefore, our findings also provide preliminary evidence for the validity of the Dutch CLQ as a measure of claustrophobia.

The predictive validity of the Dutch CLQ was examined in a second, experimental study. To this end, associations between scores of the Dutch CLQ and fear experienced in actual claustrophobic situations were explored. The pattern of results indicates that selfreported fear and respiratory reactivity (as reflected in decreases in end-tidal Fractional $\mathrm{CO}_{2}$ ) during claustrophobic exposure are related to individuals' scores on the Dutch CLQ. There were positive associations between the total CLQ score and self-reported fear during situations in which both restriction and fear of suffocation were prominent, and between the restriction scale and subjective fear during situations with restriction only. The latter finding contrasts somehow with findings from McGlynn et al. (2007) who reported that fear of restriction did not significantly contribute in path models of subjective fear during exposure to a mock scanner environment. Interestingly, scores on the restriction, but not those on the suffocation scale correlated significantly with self-reported fear during exposure to situations with risk of suffocation only. The origin of this finding is unclear, but it may relate to the specifics of the employed operationalizations. For example, an implicit social demand of the experimenter expecting somehow the participant to complete the entire experiment may have constituted an element of 'restriction', even in the suffocation-only situations. Therefore, we recommend future studies to include a manipulation check to see whether the exposure situations actually trigger the claustrophobic fear component(s) they intended to trigger.

Whereas several studies found that the CLQ did not predict cardiac responses during actual claustrophobic exposure (e.g., McGlynn et al., 2003; McGlynn, Smitherman, \& Mulfinger, 2006), the present study found that scores on the Dutch CLQ were associated with a stronger decrease in $\mathrm{FetCO}_{2}$ during exposure to claustrophobic situations. This is a rather surprising finding, given that no to very low associations between self-reports of fear and physiological reactivity are very common (Rachman \& Hodgson, 1974). A speculative explanation for the presently observed concordance between self-reports and reactivity in $\mathrm{FetCO}_{2}$ may be that a tendency towards hyperventilation may be an evolutionary 'prepared' response to claustrophobic situations (Van Diest et al., 2005).
A limitation of the present work is the small number of participants in study 2. Particularly the findings on reactivity in $\mathrm{FetCO}_{2}$ may be underpowered, so that potentially low to moderate correlations between reactivity in $\mathrm{FetCO}_{2}$ with each of the CLQ subscales may have remained undetected. Other limitations include the relative lack of men in our sample and the absence of test-retest reliability evidence.

In summary, the Dutch CLQ seems a reliable and valid instrument to measure self-reported claustrophobic fear. Similar to the English and French versions of the CLQ (Radomsky et al., 2001, 2006), it consists of two subscales, referring to restriction fear and fear of suffocation. Whereas the predictive validity of the total score of the Dutch CLQ is good both for self-reported fear and respiratory reactivity, the predictive validity of the restriction and suffocation subscales seems much weaker. More research is needed to further investigate the predictive, convergent, and discriminant validity of both subscales.

\section{References}

Arindell, W. A. (1980). Dimensional structure and psychopathology correlates of the Fear Survey Schedule (FSS-III) in a phobic situation: factorial definition of agoraphobia. Behaviour Research \& Therapy, 18, 229-242.

Barenes, L. L. B., Harp, D., \& Jung, W. S. (2002). Reliability generalization of scores on the Spielberger state-Trait anxiety inventory. Educational and Psychological Measurement, 62, 603-618.

Beck, A. T., Steer, R. A., \& Brown, G. K. (1996). Manual for the Beck Depression Inventory. San Antonio, TX: Psychological Corporation.

Bentler, P. M. (2007). On tests and indices for evaluating structural models. Personality and Individual Differences, 42, 825-829.

De Clerck, A., Verschuere, B., Crombez, G., \& De Vlieger, P. (2006). Psychophysiological analysis (PSPHA): a modular script based program for analyzing psychophysiological data. Behavior Research Methods, 38, 504-510.

Eifert, G. H., Zvolensky, M. J., Sorrell, J. T., Hopko, D. R., \& Lejuez, C. W. (1999). Predictors of self-reported anxiety and panic symptoms: an evaluation of anxiety sensitivity, suffocation fear, heart-focused anxiety, and breath-holding duration. Journal of Psychopathology and Behavioral Assessment, 21(4), 293305.

Eke, M., \& McNally, R. J. (1996). Anxiety sensitivity, suffocation fear, trait anxiety, and breath-holding duration as predictors of response to carbon dioxide challenge. Behaviour Research and Therapy, 34(8), 603-607.

Flora, D. B., \& Curran, P. J. (2004). An empirical evaluation of alternative methods of estimation for confirmatory factor analysis with ordinal data. Psychological Methods, 9, 466-491.

Gardner, W. N. (1996). The pathophysiology of hyperventilation disorders. Chest, 109(2), 516-534.

Harris, L. M., Robinson, J., \& Menzies, R. G. (1999). Evidence for fear of restriction and fear of suffocation as components of claustrophobia. Behaviour Research and Therapy, 37, 155-159.

Hoekstra, H. A., Ormel, J., \& De Fruyt, F. (1996). NEO persoonlijkheidsvragenlijsten NEO-PI-R en NEO-FFI. Handleiding [NEO personality questionnaires NEO-PI_R and NEO-FFI. Manual]. Lisse: Swets \& Zeitlinger.

Hu, L., \& Bentler, P. M. (1999). Cutoff criteria for fit indexes in covariance structure analysis: conventional criteria versus new alternatives. Structural Equation Modeling, 6, 1-55.

Jöreskog, K., \& Sörbom, D. (2004). LISRELL 8.71 [Computer Program]. Lincolnwood, IL: Scientific Software International.

McGlynn, F. D., Karg, R., \& Lawyer, S. R. (2003). Fear responses to mock magnetic resonance imaging among college students: toward a prototype experiment. Journal of Anxiety Disorders, 17(3), 335-347.

McGlynn, F. D., Smitherman, T. A., Hammel, J. C., \& Lazarte, A. A. (2007). Component fears of claustrophobia associated with mock magnetic resonance imaging. Journal of Anxiety Disorders, 21, 367-380.

McGlynn, F. D., Smitherman, T. A., \& Mulfinger, A. M. M. (2006). Anxiety and fear. In: M. Hersen (Ed.), Clinician's handbook of adult behavioral assessment (pp. 189-208). Burlington: Academic Press.

McIsaac, H. K., Thordarson, D. S., Shafran, R., Rachman, S., \& Poole, P. (1998). Claustrophobia and the magnetic resonance imaging procedure. Journal of Behavioral Medicine, 21(3), 255-268.

McNeil, D. W., \& Rainwater, A. J. (1998). Development of the Fear of Pain Questionnaire-III. Journal of Behavioral Medicine, 21, 389-410.

Öst, L.-G. (2007). The claustrophobia scale: a psychometric evaluation. Behaviour Research and Therapy, 45, 1053-1064.

Rachman, S. J. (1997). Claustrophobia. In: G. C. L. Davey (Ed.), Phobias: a handbook of theory, research and treatment (pp. 163-181). Brighton, UK: University of Sussex.

Rachman, S., \& Hodgson, R. (1974). Synchrony and desynchrony in fear and avoidance. Behaviour Research and Therapy, 12(4), 311-318.

Rachman, S., \& Taylor, S. (1993). Analyses of claustrophobia. Journal of Anxiety Disorders, 7, 281-291. 
Radomsky, A. S., Quimet, A. J., Ashbaugh, A. R., Paradis, M. R., Lavoie, S. L., \& O'Conner, K. P. (2006). Psychometric properties of the French and English versions of the Claustrophobia Questionnaire (CLQ). Anxiety Disorders, 20, 818-828.

Radomsky, A. S., Rachman, S., Thordarson, D. S., McIsaak, H. K., \& Teachman, B. A. (2001). The claustrophobia questionnaire. Journal of Anxiety Disorders, 15, 287-297.

Rassovsky, Y., Kushner, M. G, Schwarze, N. J. \& Wangensteen, O. D. (2000). Psychological and physiological predictors of response to carbon dioxide challenge in individuals with panic disorder. Journal of Abnormal Psychology, 109(4), 616-623. Roelofs, J., Peters, M. L., Deutz, J., Spijker, C., \& Vlaeyen, J. W. S. (2005). The Fear of Pain Questionnaire (FPQ): further psychometric examination in a non-clinical sample. Pain, 116, 339-346.

Shipherd, J. C., Beck, J. G., \& Ohtake, P. J. (2001). Relationships between the anxiety sensitivity index, the suffocation fear scale, and responses to $\mathrm{CO}_{2}$ inhalation. Journal of Anxiety Disorders, 15(3), 247-258.

Spielberger, C. D. (1983). Manual for the State-Trait Anxiety Inventory (Form Y). Palo Alto, CA: Mind Garden.

Taylor, S., Zvolensky, M. J., Cox, B. J., Deacon, B., Heimberg, R. G., Ledley, D. R., et al. (2007). Robust dimensions of anxiety sensitivity development and initial validation of the Anxiety Sensitivity Index - 3. Psychological Assessment, 19 $176-188$.

van der Droes, A. J. W. (2002). BDI-II-NL: Nederlandse vertaling en bewerking [BDIII-NL: The Dutch translation and revision]. Enschede, The Netherlands: The Psychological Corporation.

van der Ploeg, H. M. (2000). Een Nederlandstalige bewerking van de Spielberger StateTrait Anxiety Inverntory: STAI-DY [A Dutch revision of the Spielberger State-Trait Anxiety Inventory: STAI-DY]. Lisse, The Netherlands: Swets Test Publishers.

Van Diest, I., De Peuter, S., Devriese, S., Wellens, E., Van de Woestijne, K. P., \& Van den Bergh, O. (2005). Imagined risk of suffocation as a trigger for hyperventilation. Psychosomatic Medicine, 67, 813-819.

Van Diest, I., Winters, W., Devriese, S., Vercamst, E., Han, J. N., Van de Woestijne K. P., et al. (2001). Hyperventilation beyond fight/flight: respiratory responses during emotional imagery. Psychophysiology, 38, 961-968.

Wolpe, J., \& Lang, P. J. (1964). A Fear Survey Schedule for use in behavior therapy. Behaviour Research and Therapy, 2, 27-30. 